\title{
Exercise Therapy for Patients With Type 2 Diabetes: A Narrative Review
}

\author{
Hidekatsu Yanai a, b, Hiroki Adachia, Yoshinori Masui ${ }^{a}$, Hisayuki Katsuyama ${ }^{a}$, \\ Akiko Kawaguchi $^{\text {a }}$, Mariko Hakoshima ${ }^{a}$, Yoko Waragai ${ }^{a}$, Tadanao Harigae ${ }^{a}$, \\ Hidetaka Hamasakia ${ }^{\text {a }}$ Akahito Sako ${ }^{a}$
}

\begin{abstract}
To achieve excellent glycemic control in patients with type 2 diabetes, an adequate prescription of exercise therapy is required. The meta-analyses proposed that high-intensity training improves metabolic parameters in patients with pre-diabetes or type 2 diabetes and low physical activity is associated with an increased risk of incident type 2 diabetes. Here, we would introduce literatures about effects of physical activity on mortality, cardiovascular events, and metabolic parameters, to encourage understanding of exercise therapy, and then describe how to prescribe exercise therapy for patients with type 2 diabetes. We also show the usefulness of non-exercise activity thermogenesis for diabetic patients who cannot perform volitional sportinglike exercise because of diabetic complication and/or comorbidity, by presenting results of our previous studies.
\end{abstract}

Keywords: Exercise; Insulin sensitivity; Non-exercise activity thermogenesis; Type 2 diabetes

\section{Introduction}

To achieve good glycemic control in patients with type 2 diabetes, an adequate exercise therapy is required. The meta-analyses reported that high-intensity training ameliorates metabolic parameters in individuals at risk of or with type 2 diabetes, and that low physical activity (PA) doubles an increased risk of incident type 2 diabetes as compared with high PA [1,2]. Here, we would introduce literatures about effects of PA on mortality, cardiovascular events, and metabolic parameters, to encourage understanding of exercise therapy, and then describe how to prescribe exercise therapy for patients with

Manuscript submitted February 9, 2018, accepted February 26, 2018

a Department of Internal Medicine, National Center for Global Health and Medicine Kohnodai Hospital, Chiba, Japan

${ }^{b}$ Corresponding Author: Hidekatsu Yanai, Department of Internal Medicine, National Center for Global Health and Medicine Kohnodai Hospital, 1-7-1 Kohnodai, Ichikawa, Chiba 272-8516, Japan.

Email: dyanai@hospk.ncgm.go.jp

doi: https://doi.org/10.14740/jocmr3382w diabetes. We also present the effects of non-exercise activity thermogenesis (NEAT), which is the energy expenditure of all physical activities other than volitional sporting-like exercise [3], on metabolic parameters, in patients with diabetes and prediabetes, by using our previous studies.

\section{Types of Exercise}

The American College of Sports Medicine (ACSM) defines aerobic exercise as any activity that uses large muscle groups, can be maintained continuously and is rhythmic in nature [4]. Muscles activated by aerobic exercise rely on aerobic metabolism to extract energy in the form of adenosine triphosphate (ATP) from amino acids, carbohydrates and fatty acids. Aerobic exercises include cycling, dancing, jogging, swimming and walking. Resistance exercise is included in anaerobic exercise defined by the ACSM as intense PA of very short duration, fueled by the energy sources within the contracting muscles and independent of the use of inhaled oxygen as an energy source [5]. Without the use of oxygen, ATP is produced by anaerobic glycolysis. Resistance training causes the muscles to contract against an external resistance with the expectation of increases in strength, tone, mass, and/or endurance, and includes power-lifting, training using dumbbells and barbells. The systematic review and meta-analysis showed that resistance training improved measures of balance and muscle strength/power even in older adults [6].

\section{Effects of Exercise on Mortality and Cardiovas- cular Events}

Exercise increases cardiorespiratory fitness (CRF) [7]. Epidemiological studies have indicated an inverse association of CRF with the incidence of coronary heart disease (CHD), cardiovascular disease (CVD) or mortality, in healthy participants. The systematic review including 33 eligible studies showed relative risk (RR) of all-cause mortality and CHD/ CVD events per 1 - metabolic equivalent (MET) higher level of maximal aerobic capacity were 0.87 (95\% confidence interval (CI), $0.84-0.90)$ and 0.85 (95\% CI, 0.82 - 0.88), respectively, suggesting that better CRF was associated with lower risk of all-cause mortality and CHD/CVD [8]. To understand 
the association between walking and the risk for all-cause and CVD mortality among persons with diabetes $(n=2,896$, age $\geq$ 18 years), a prospective cohort study was performed [9]. Compared with inactive individuals, those who walked at least 2 $\mathrm{h} /$ week had a $39 \%$ lower all-cause mortality rate and a $34 \%$ lower CVD mortality rate [9]. The mortality rates were lowest for persons who walked 3 - $4 \mathrm{~h} /$ week (all-cause mortality hazard ratio (HR), 0.46; 95\% CI, 0.29 - 0.71; CVD mortality HR, $0.47 ; 95 \% \mathrm{CI}, 0.24-0.91)$ and for those who reported that their walking involved moderate increases in heart and breathing rates (all-cause mortality HR, 0.57; 95\% CI, $0.41-0.80$; CVD mortality HR, 0.69; 95\% CI, 0.43 - 1.09) [9].

The meta-analysis which evaluated the CVD risk reduction in relation to PA, focusing on clarifying dose-response associations, was performed [10]. There were 17 eligible studies. The highest PA category had a lower RR for all-cause mortality $(0.61(0.52-0.70))$ and CVD $(0.71(0.60-0.84))$ than the lowest PA category. A one MET-h/day incrementally higher PA was associated with $9.5 \%$ and $7.9 \%$ reductions in all-cause mortality and CVD risk, respectively.

\section{Effects of Exercise on Metabolic Parameters}

\section{Effects on glucose metabolism}

A systematic review and meta-analysis of 47 randomized controlled clinical trials (RCTs) assessing associations of structured exercise training regimens and PA advice on change in hemoglobin A1c (HbA1c) in type 2 diabetic patients was conducted [11]. Structured exercise training was associated with a decline in HbA1c level (-0.67\%) compared with control participants. Structured aerobic exercise $(-0.73 \%)$, structured resistance training (RT) $(-0.57 \%)$, and both combined $(-0.51 \%)$ were associated with declines in HbAlc levels compared with control. Structured exercise durations of more than $150 \mathrm{~min} /$ week were associated with $\mathrm{HbA} 1 \mathrm{c}$ reduction of $0.89 \%$, while structured exercise durations of $150 \mathrm{~min}$ or less per week were associated with $\mathrm{HbA} 1 \mathrm{c}$ reduction of $0.36 \%$. PA advice alone was not associated with the changes of HbAlc.

In a systematic review of $27 \mathrm{RCT}$ s assessing the association between intensity and volume of exercise training and $\mathrm{HbA} 1 \mathrm{c}$ changes in patients with type 2 diabetes, aerobic exercise volume (frequency of sessions) was associated with changes in HbA1c [12]. In combined training, weekly volume of RT was associated with reduction in HbA1c levels.

Supervised RT improved glycemic control and insulin sensitivity; however, when supervision was removed compliance and glycemic control decreased [13]. To examine the effect of RT combined with moderate weight loss (WL) on glycemic control and body composition in older patients with type 2 diabetes, sedentary, overweight men and women with type 2 diabetes, aged $60-80$ years $(n=36)$, were randomized to RT plus WL or WL plus a control program for 6 months [14]. HbA1c fell significantly more in RT and WL than WL at 3 months $(0.6 \pm 0.7$ vs. $0.07 \pm 0.8 \%, \mathrm{P}<0.05)$ and 6 months $(1.2 \pm 1.0$ vs. $0.4 \pm 0.8 \%, \mathrm{P}<0.05)$. Similar reductions in body weight and fat mass were observed after 6 months. In contrast, lean body mass increased in the RT and WL group $(0.5 \pm 1.1$ $\mathrm{kg})$ and decreased in the WL group $(0.4 \pm 1.0)$ after 6 months $(\mathrm{P}<0.05)$.

In another RCT, among older adults with type 2 diabetes, 16 weeks of RT (three times per week) resulted in reduced $\mathrm{HbA} 1 \mathrm{c}$ (from $8.7 \pm 0.3$ to $7.6 \pm 0.2 \%$ ), and reduced the dose of prescribed anti-diabetic medication in $72 \%$ of exercisers compared with the control group [15]. RT increased lean mass $(+1.2 \pm 0.2$ vs. $-0.1 \pm 0.1 \mathrm{~kg})$, reduced systolic blood pressure $(-9.7 \pm 1.6$ vs. $+7.7 \pm 1.9 \mathrm{~mm} \mathrm{Hg})$, and decreased trunk fat mass $(-0.7 \pm 0.1$ vs. $+0.8 \pm 0.1 \mathrm{~kg})$ as compared with the control group.

Using continuous glucose monitoring (CGM), Gillen et al examined the 24-h blood glucose response to one session of high-intensity interval training (HIT) consisting of $10 \times 60 \mathrm{~s}$ cycling efforts at about $90 \%$ maximal heart rate, interspersed with $60 \mathrm{~s}$ rest [16]. HIT reduced hyperglycemia measured as proportion of time spent above $10 \mathrm{mmol} / \mathrm{L}$ (HIT: $4.5 \pm 4.4 \mathrm{vs}$. control: $15.2 \pm 12.3 \%, \mathrm{P}=0.04)$. Postprandial hyperglycemia, measured as the sum of post-meal areas under the glucose curve, was also lower after HIT as compared with control (728 \pm 331 vs. $1,142 \pm 556 \mathrm{mmol} / \mathrm{L} \times 9 \mathrm{~h}, \mathrm{P}=0.01)$.

Eight patients with type 2 diabetes $(63 \pm 8$ years, BMI $32 \pm$ $6 \mathrm{~kg} / \mathrm{m}^{2}, \mathrm{HbA} 1 \mathrm{c} 6.9 \pm 0.7 \%$ ) performed six sessions of HIT (10 $\times 60$ s cycling bouts eliciting about $90 \%$ maximal heart rate, interspersed with $60 \mathrm{~s}$ rest) over 2 weeks [17]. Average 24-h blood glucose concentration was reduced after training from $7.6 \pm 1.0$ to $6.6 \pm 0.7 \mathrm{mmol} / \mathrm{L}$.

In the systematic review which examined the effect of exercise as assessed by CGM in type 2 diabetes, exercise significantly decreased average glucose concentrations $(-0.8 \mathrm{mmol} / \mathrm{L}$, $\mathrm{P}<0.01)$ and daily time spent in hyperglycemia $(-129 \mathrm{~min}$, $\mathrm{P}<0.01)$, but did not significantly affect daily time spent in hypoglycemia $(-3 \mathrm{~min}, \mathrm{P}=0.47)$ or fasting glucose $(-0.3 \mathrm{mmol} / \mathrm{L}$, $\mathrm{P}=0.13)[18]$.

\section{Effects on adiposity, lipid metabolism, blood pressure and inflammatory cytokines}

In a meta-analysis of $12 \mathrm{RCTs}$ which examined the effects of 8 weeks or more of aerobic exercise on lipids and lipoproteins in adults with type 2 diabetes, a statistically significant reduction of about $5 \%$ was found for low-density lipoprotein cholesterol (LDL-C) [19]. The meta-analysis of 14 RCTs showed that exercise significantly improves glycemic control and reduces visceral adipose tissue and plasma triglycerides, but not plasma cholesterol, in people with type 2 diabetes, even without weight loss [20].

In the meta-analysis of $42 \mathrm{RCTs}$, structured exercise was associated with a change in systolic blood pressure of -2.42 $\mathrm{mm} \mathrm{Hg}$, diastolic blood pressure of $-2.23 \mathrm{~mm} \mathrm{Hg}$, high-density lipoprotein cholesterol (HDL-C) of $+0.04 \mathrm{mmol} / \mathrm{L}$, and LDL$\mathrm{C}$ of $-0.16 \mathrm{mmol} / \mathrm{L}$ [21]. In the meta-analysis of $14 \mathrm{RCTs}$, exercise was associated with a significant $\mathrm{C}$-reactive protein (CRP) of $-0.66 \mathrm{mg} / \mathrm{L}$ and interleukin-6 (IL-6) of $-0.88 \mathrm{pg} / \mathrm{mL}$ [22]. Aerobic exercise program was associated with a significant change in leptin of $-3.72 \mathrm{ng} / \mathrm{mL}$. 


\section{The Differences in Effects on Metabolic Param- eters Between Aerobic Exercise and Resistance Exercise}

To determine whether aerobic plus resistance training (AT + RT) is more beneficial than aerobic training (AT) for metabolic parameters, a total of 139 obese adolescents were randomized into two groups: AT $(n=55)$, AT + RT $(n=61)$ [23]. The AT + RT group showed better results with regard to decreased body fat mass, LDL-C levels, subcutaneous and visceral fat and increased body lean mass as compared with AT alone. A total of 30 adolescents (aged 15 - 19 years, BMI $\geq 95$ percentile) were divided into two groups: AT $(\mathrm{n}=15)$ and AT + RT $(\mathrm{n}=$ 15) [24]. The AT + RT group had significantly higher changes throughout the intervention in body composition, total cholesterol, waist circumference, glucose, and adiponectin than AT alone.

In the systematic review with meta-analysis (12 trials with 555 youths), compared with AT alone, concurrent exercise plus $\mathrm{RT}$ resulted in greater reductions in body mass $(-2.28 \mathrm{~kg})$, fat mass $(-3.49 \%)$ and LDL-C $(-10.20 \mathrm{mg} / \mathrm{dL})$, as well as greater increases in lean body mass $(+2.20 \mathrm{~kg})$ and adiponectin level $(+2.59 \mu \mathrm{g} / \mathrm{mL})[25]$.

Forty-eight patients with type 2 diabetes were randomly assigned to four groups of 12-week training: AT group $(\mathrm{n}=$ $12)$, RT group $(\mathrm{n}=12)$, combine group $(\mathrm{n}=12)$, and control group $(n=12)$ [26]. All four groups displayed decreases in blood pressure, fasting plasma glucose, postprandial plasma glucose, lipid profile, and high-sensitivity CRP $(\mathrm{P}<0.05)$, and there was no difference across the groups. Thirty obese women, aged between 35 and 45 years with BMI of above 30 were grouped into control $(\mathrm{n}=10)$, AT $(\mathrm{n}=10)$ and RT $(\mathrm{n}=$ 10) [27]. Significant differences were observed in very lowdensity lipoprotein (VLDL) (from $28.1 \pm 1.4$ to $26.9 \pm 0.8 \mathrm{mg}$ / $\mathrm{dL}$ ) and HDL (from $45.4 \pm 3.5$ to $53.6 \pm 3.1 \mathrm{mg} / \mathrm{dL}$ ) levels in AT group with $\mathrm{P}<0.001$. A total of 60 obese women with severe eating disorders were randomly divided into three groups: control group with no exercise $(n=20)$, AT group $(n=20)$, and RT group $(n=20)$ [28]. Reduced LDL-C level, fat mass, homeostasis model assessment (HOMA-IR) measurements were observed only in AT.

In the meta-analysis including 15 trials enrolling 741 participants, compared to RT, AT resulted in a significantly more pronounced reduction of body weight $(-1.15 \mathrm{~kg})$, waist circumference $(-1.10 \mathrm{~cm})$, and fat mass $(-1.15 \mathrm{~kg})$, respectively. RT was more effective than AT in improving lean body mass $(+1.26 \mathrm{~kg})$ [29].

\section{How to Prescribe Exercise Therapy for Patients With Diabetes}

When we prescribe exercise therapy for patients with diabetes, we have to present the following items: 1) kinds of exercise (aerobic exercise or anaerobic exercise including RT), 2) intensity of exercise (METs, etc.), 3) frequency (how many times per week), and 4) duration (how long per day or week).
According to the recommendation by American Diabetes Association [30], adults with diabetes should be advised to perform at least $150 \mathrm{~min} /$ week of moderate-intensity aerobic PA (50-70\% of maximum heart rate), spread over at least 3 days/ week with no more than 2 consecutive days without exercise. In the absence of contraindications, adults with type 2 diabetes should be encouraged to perform RT at least twice per week.

\section{NEAT}

The concept of NEAT was presented by Levine [3]. NEAT is the energy expenditure of all physical activities other than volitional sporting-like exercise [3]. NEAT includes all the activities that render us vibrant, unique, and independent beings such as working, playing and dancing.

We developed the original questionnaire to evaluate NEAT in Japanese people, based on 2011 Compendium of Physical Activities produced by American College of Sports Medicine [31], by considering Japanese life-style and culture [32]. The questionnaire consisted of 11 question items about locomotive activities and 25 question items about non-locomotive activities [32]. We evaluated each questionnaire item with a score of 1 to 3 points in order of levels of daily PA and then added up the scores to determine the NEAT score.

The NEAT score was negatively correlated with serum insulin levels $(\mathrm{r}=-0.42, \mathrm{P}<0.05)$ in all subjects [32]. The NEAT score was also negatively correlated with waist circumference $(\mathrm{r}=-0.509, \mathrm{P}<0.05)$ and positively correlated with HDL-C levels $(\mathrm{r}=0.494, \mathrm{P}<0.05)$ in women, and was negatively associated with serum insulin levels $(\mathrm{r}=-0.732, \mathrm{P}<0.005)$, systolic $(\mathrm{r}=-0.482, \mathrm{P}<0.05)$ and diastolic blood pressure $(\mathrm{r}=$ $-0.538, \mathrm{P}<0.05)$ in patients with abdominal obesity [32]. Our study demonstrated that NEAT is associated with amelioration in insulin sensitivity, waist circumference, HDL-C, blood pressure, in patients with type 2 diabetes [32].

We examined the validity of our NEAT questionnaire by comparing with objectively measured daily PA by using the triaxial accelerometer [33]. The NEAT score was significantly and positively correlated with PA level (PAL) measured by the triaxial accelerometer $(\mathrm{r}=0.604, \mathrm{P}<0.001)$. PAL was also significantly and positively correlated with both the locomotive NEAT score and the non-locomotive NEAT score $(r=0.444$, $\mathrm{P}=0.001$ and $\mathrm{r}=0.526, \mathrm{P}<0.001$, respectively). The NEAT score measured by the self-reported questionnaire was highly correlated with PAL measured by the triaxial accelerometer.

In type 2 diabetic women, the NEAT score was significantly and negatively correlated with urinary albumin creatinine ratio $(\mathrm{UACR})(\mathrm{r}=-0.513, \mathrm{P}<0.05)$ and positively correlated with coefficient of variation of R-R intervals (CVRR) $(r=0.471, P<0.05)$ [34]. Our study suggested that NEAT is associated with markers for diabetic nephropathy and neuropathy in type 2 diabetic women.

\section{Conclusion}

We summarized and showed beneficial effects of PA includ- 
Table 1. Beneficial Effects of Physical Activity Including Aerobic Exercise, Resistant Training and Non-Exercise Activity Thermogenesis [1, 2, 6-29, 32, 34]

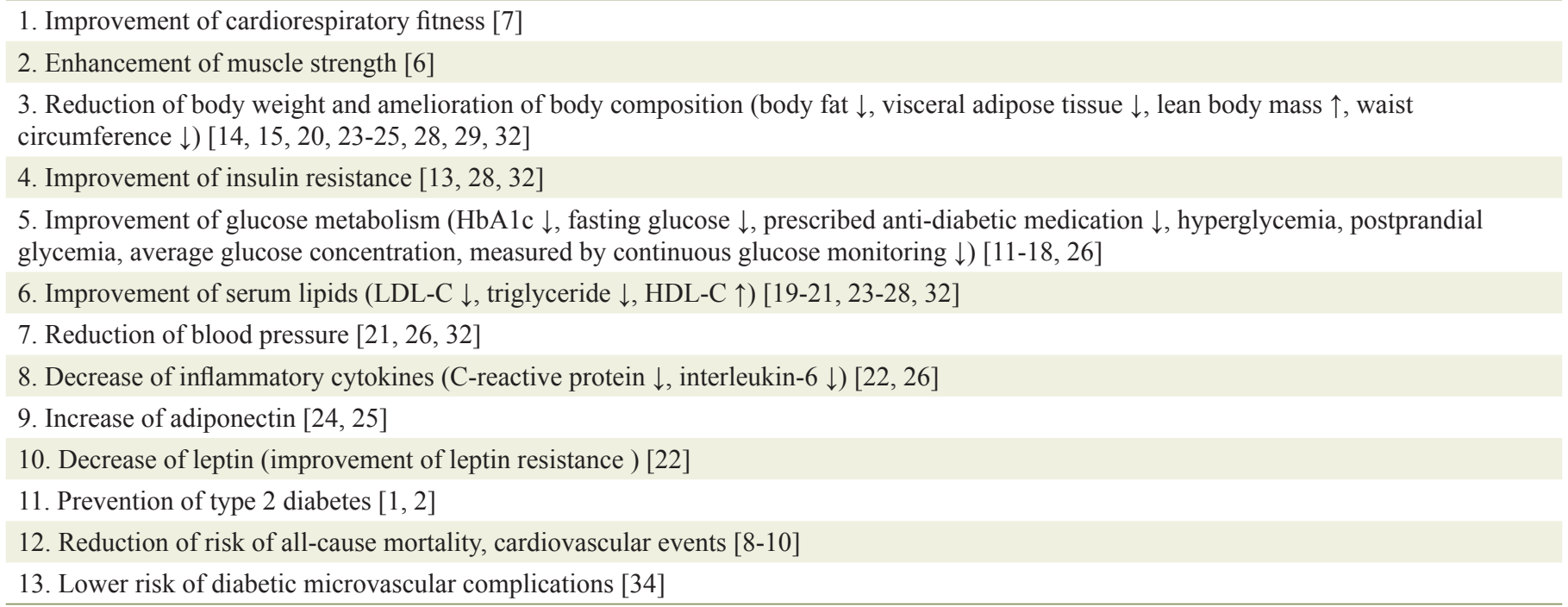

ing aerobic exercise, RT and NEAT in Table 1 [1, 2, 6-29, 32, 34]. An increase of PA improves cardiorespiratory fitness and muscle strength, and insulin resistance, glucose and lipid metabolism, and reduces body weight and fat, blood pressure, inflammatory cytokines and leptin. High PA is associated with lower incidence of type 2 diabetes, mortality and cardiovascular events.

\section{Competing Interests}

The authors declare that they have no competing interests concerning this article.

\section{References}

1. Jelleyman C, Yates T, O’Donovan G, Gray LJ, King JA, Khunti K, Davies MJ. The effects of high-intensity interval training on glucose regulation and insulin resistance: a meta-analysis. Obes Rev. 2015;16(11):942-961.

2. Cloostermans L, Wendel-Vos W, Doornbos G, Howard B, Craig CL, Kivimaki M, Tabak AG, et al. Independent and combined effects of physical activity and body mass index on the development of Type 2 Diabetes - a metaanalysis of 9 prospective cohort studies. Int J Behav Nutr Phys Act. 2015;12:147.

3. Levine JA, Vander Weg MW, Hill JO, Klesges RC. Nonexercise activity thermogenesis: the crouching tiger hidden dragon of societal weight gain. Arterioscler Thromb Vasc Biol. 2006;26(4):729-736.

4. Wahid A, Manek N, Nichols M, Kelly P, Foster C, Webster P, Kaur A, et al. Quantifying the Association Between Physical Activity and Cardiovascular Disease and Diabetes: A Systematic Review and Meta-Analysis. J Am Heart Assoc. 2016;5(9):e002495.
5. American College of Sports Medicine. ACSM's guidelines for exercise testing and prescription. USA: Lippincott Williams \& Wilkins; 2013.

6. Lacroix A, Hortobagyi T, Beurskens R, Granacher U. Effects of supervised vs. unsupervised training programs on balance and muscle strength in older adults: a systematic review and meta-analysis. Sports Med. 2017;47(11):23412361.

7. Segal KR, Pi-Sunyer FX. Exercise and obesity. Med Clin North Am. 1989;73(1):217-236.

8. Kodama S, Saito K, Tanaka S, Maki M, Yachi Y, Asumi M, Sugawara A, et al. Cardiorespiratory fitness as a quantitative predictor of all-cause mortality and cardiovascular events in healthy men and women: a meta-analysis. JAMA. 2009;301(19):2024-2035.

9. Gregg EW, Gerzoff RB, Caspersen CJ, Williamson DF, Narayan KM. Relationship of walking to mortality among US adults with diabetes. Arch Intern Med. 2003;163(12):1440-1447.

10. Kodama S, Tanaka S, Heianza Y, Fujihara K, Horikawa C, Shimano H, Saito K, et al. Association between physical activity and risk of all-cause mortality and cardiovascular disease in patients with diabetes: a meta-analysis. Diabetes Care. 2013;36(2):471-479.

11. Umpierre D, Ribeiro PA, Kramer CK, Leitao CB, Zucatti AT, Azevedo MJ, Gross JL, et al. Physical activity advice only or structured exercise training and association with HbA1c levels in type 2 diabetes: a systematic review and meta-analysis. JAMA. 2011;305(17):1790-1799.

12. Umpierre D, Ribeiro PA, Schaan BD, Ribeiro JP. Volume of supervised exercise training impacts glycaemic control in patients with type 2 diabetes: a systematic review with meta-regression analysis. Diabetologia. 2013;56(2):242251.

13. Gordon BA, Benson AC, Bird SR, Fraser SF. Resistance training improves metabolic health in type 2 dia- 
betes: a systematic review. Diabetes Res Clin Pract. 2009;83(2):157-175.

14. Dunstan DW, Daly RM, Owen N, Jolley D, De Courten M, Shaw J, Zimmet P. High-intensity resistance training improves glycemic control in older patients with type 2 diabetes. Diabetes Care. 2002;25(10):1729-1736.

15. Castaneda C, Layne JE, Munoz-Orians L, Gordon PL, Walsmith J, Foldvari M, Roubenoff R, et al. A randomized controlled trial of resistance exercise training to improve glycemic control in older adults with type 2 diabetes. Diabetes Care. 2002;25(12):2335-2341.

16. Gillen JB, Little JP, Punthakee Z, Tarnopolsky MA, Riddell MC, Gibala MJ. Acute high-intensity interval exercise reduces the postprandial glucose response and prevalence of hyperglycaemia in patients with type 2 diabetes. Diabetes Obes Metab. 2012;14(6):575-577.

17. Little JP, Gillen JB, Percival ME, Safdar A, Tarnopolsky MA, Punthakee Z, Jung ME, et al. Low-volume highintensity interval training reduces hyperglycemia and increases muscle mitochondrial capacity in patients with type 2 diabetes. J Appl Physiol (1985). 2011;111(6):15541560.

18. MacLeod SF, Terada T, Chahal BS, Boule NG. Exercise lowers postprandial glucose but not fasting glucose in type 2 diabetes: a meta-analysis of studies using continuous glucose monitoring. Diabetes Metab Res Rev. 2013;29(8):593-603.

19. Kelley GA, Kelley KS. Effects of aerobic exercise on lipids and lipoproteins in adults with type 2 diabetes: a meta-analysis of randomized-controlled trials. Public Health. 2007;121(9):643-655.

20. Thomas DE, Elliott EJ, Naughton GA. Exercise for type 2 diabetes mellitus. Cochrane Database Syst Rev. 2006;3:CD002968.

21. Hayashino Y, Jackson JL, Fukumori N, Nakamura F, Fukuhara S. Effects of supervised exercise on lipid profiles and blood pressure control in people with type 2 diabetes mellitus: a meta-analysis of randomized controlled trials. Diabetes Res Clin Pract. 2012;98(3):349-360.

22. Hayashino Y, Jackson JL, Hirata T, Fukumori N, Nakamura F, Fukuhara S, Tsujii S, et al. Effects of exercise on C-reactive protein, inflammatory cytokine and adipokine in patients with type 2 diabetes: a meta-analysis of randomized controlled trials. Metabolism. 2014;63(3):431440.

23. Damaso AR, da Silveira Campos RM, Caranti DA, de Piano A, Fisberg M, Foschini D, de Lima Sanches P, et al. Aerobic plus resistance training was more effective in improving the visceral adiposity, metabolic profile and inflammatory markers than aerobic training in obese adolescents. J Sports Sci. 2014;32(15):1435-1445.

24. de Mello MT, de Piano A, Carnier J, Sanches Pde L, Cor- rea FA, Tock L, Ernandes RM, et al. Long-term effects of aerobic plus resistance training on the metabolic syndrome and adiponectinemia in obese adolescents. J Clin Hypertens (Greenwich). 2011;13(5):343-350.

25. Garcia-Hermoso A, Ramirez-Velez R, Ramirez-Campillo R, Peterson MD, Martinez-Vizcaino V. Concurrent aerobic plus resistance exercise versus aerobic exercise alone to improve health outcomes in paediatric obesity: a systematic review and meta-analysis. Br J Sports Med. 2018;52(3):161-166.

26. Jorge ML, de Oliveira VN, Resende NM, Paraiso LF, Calixto A, Diniz AL, Resende ES, et al. The effects of aerobic, resistance, and combined exercise on metabolic control, inflammatory markers, adipocytokines, and muscle insulin signaling in patients with type 2 diabetes mellitus. Metabolism. 2011;60(9):1244-1252.

27. Chaudhary S, Kang MK, Sandhu JS. The effects of aerobic versus resistance training on cardiovascular fitness in obese sedentary females. Asian J Sports Med. 2010;1(4):177-184.

28. Fenkci S, Sarsan A, Rota S, Ardic F. Effects of resistance or aerobic exercises on metabolic parameters in obese women who are not on a diet. Adv Ther. 2006;23(3):404413.

29. Schwingshackl L, Dias S, Strasser B, Hoffmann G. Impact of different training modalities on anthropometric and metabolic characteristics in overweight/obese subjects: a systematic review and network meta-analysis. PLoS One. 2013;8(12):e82853.

30. American Diabetes A. 3. Foundations of Care and Comprehensive Medical Evaluation. Diabetes Care. 2016;39(Suppl 1):S23-35.

31. Ainsworth BE, Haskell WL, Herrmann SD, Meckes N, Bassett DR, Jr., Tudor-Locke C, Greer JL, et al. 2011 Compendium of Physical Activities: a second update of codes and MET values. Med Sci Sports Exerc. 2011;43(8):1575-1581.

32. Hamasaki H, Yanai H, Mishima S, Mineyama T, Yamamoto-Honda R, Kakei M, Ezaki O, et al. Correlations of nonexercise activity thermogenesis to metabolic parameters in Japanese patients with type 2 diabetes. Diabetol Metab Syndr. 2013;5(1):26.

33. Hamasaki H, Yanai H, Kakei M, Noda M, Ezaki O. The validity of the non-exercise activity thermogenesis questionnaire evaluated by objectively measured daily physical activity by the triaxial accelerometer. BMC Sports Sci Med Rehabil. 2014;6:27.

34. Hamasaki H, Yanai H, Kakei M, Ezaki O, Noda M. Nonexercise activity thermogenesis is associated with markers for diabetic microangiopathy in Japanese female patients with type 2 diabetes. Int J Cardiol. 2013;168(5):48364837. 